\section{Fatores relacionados ao desmame precoce do aleitamento materno}

\author{
Factors related to early weaning of breastfeeding
}

Factores relacionados al destete precoz de la lactancia materna
Heuler Souza Andrade

Raquel Aparecida Pessoa

Lívia Cristina Vasconcelos Donizete

\title{
Resumo
}

Objetivos: Investigar os fatores relacionados ao desmame precoce antes dos seis meses de vida. Métodos: Trata-se de uma pesquisa exploratória, descritiva de abordagem quantitativa, baseada em questionário semiestruturado, com amostra de 52 mulheres com filhos de 0 a 6 meses de vida que não estavam mais em aleitamento materno exclusivo (AME), pertencentes às Estratégias de Saúde da Família urbanas do município de Santo Antônio do Monte, centro-oeste de Minas Gerais. Resultados: Apontaram mães jovens, casadas, primíparas, inseguras, com gravidez não planejada, realização das consultas de pré-natal periodicamente, desmame do AME entre o quarto e quinto mês de vida da criança. Conclusão: As atividades laborais, as crenças quanto ao leite materno ser fraco ou insuficiente para o bebê e a má interpretação do choro do recém-nascido estão entre as causas encontradas para o desmame precoce. Salienta-se a implementação de estratégias e ações educativas que priorizem a resolutividade quanto aos fatores destacados, e que, especialmente, busquem melhorar o vínculo com esse público.

Palavras-chave: Aleitamento Materno; Desmame Precoce; Promoção da Saúde; Atenção Primária à Saúde; Estratégia Saúde da Família

\begin{abstract}
Objectives: To investigate the factors related to early weaning before six months of life. Methods: This is an exploratory, descriptive, quantitative approach, based on a semi-structured form carried out with 52 mothers of children from 0 to 6 months of age who were no longer in Exclusive Breastfeeding (EB), belonging to Family Health Strategies of urban centers of the municipality of Santo Antônio do Monte, midwest of Minas Gerais. Results: Most of the participants were young mothers, married, primiparous, insecure, with unplanned pregnancies, performed prenatal consultations periodically, interrupted the EB between the fourth and fifth month of the child's life. Most indicated guidance by health professionals as a facilitator for adherence to the EB. Conclusion: Labor activities, beliefs about breast milk being weak or insufficient for the baby and misinterpretation of crying of the newborn are among the causes found for early weaning. We emphasize the implementation of strategies and educational actions that prioritize the resolution of the highlighted factors, and especially, that seek to improve the bond with this public.
\end{abstract}

Keywords: Breast Feeding; Early Weaning; Health Promotion; Primary Health Care; Family Health Strategy
Universidade do Estado de Minas Gerais (UEMG). Divinópolis, MG, Brasil. heulerandrade@gmail.com (Autor correspondente); raquelpessoa09@gmail.com; vlivia@hotmail.com
Como citar: Andrade HS, Pessoa RA, Donizete LCV. Fatores relacionados ao desmame precoce do aleitamento materno. Rev Bras Med Fam Comunidade. 2018;13(40):1-11. http://dx.doi.org/10.5712/rbmfc13(40)1698
Fonte de financiamento: declaram não haver.

Parecer CEP: 2.086.812 (UEMG), aprovado em 29/05/2017.

Conflito de interesses: declaram não haver. Procedência e revisão por pares: revisado por pares. Recebido em: 23/02/2018. Aprovado em: 17/05/2018. 


\section{Resumen}

Objetivos: Investigar los factores relacionados con el destete precoz antes de los seis meses de vida. Métodos: Se trata de una investigación exploratoria, descriptiva de abordaje cuantitativo, basado en cuestionario semiestructurado, con muestra de 52 mujeres con hijos de 0 a 6 meses de vida que no estaban más en lactancia materna exclusiva (LME), pertenecientes a las Estrategias de Salud de la Familia urbanas del municipio de Santo Antônio do Monte, centro oeste de Minas Gerais. Resultados: Se indicaron madres jóvenes, casadas, primíparas, inseguras, con embarazo no planificado, realización de las consultas de prenatal periódicamente, destete del LME entre el cuarto y quinto mes de vida del niño. Conclusión: Las actividades laborales, las creencias de que la leche materna es débil o insuficiente para el bebé y la mala interpretación del llanto del recién nacido están entre las causas encontradas para el destete precoz. Se destaca la implementación de estrategias y acciones educativas que prioricen la resolutividad en cuanto a los factores destacados, y que, especialmente, busquen mejorar el vínculo con ese público.

Palabras clave: Lactancia Materna; Destete Precoz; Promoción de la Salud; Atención Primaria de Salud; Estrategias de Salud Familiar

\section{Introdução}

A Organização Mundial de Saúde (OMS) e o Ministério da Saúde (MS) recomendam que os recém-nascidos $(R N)$ recebam aleitamento materno $(A M)$ até os 2 anos de idade, e o aleitamento materno exclusivo (AME) até os 6 meses de vida. Amamentar não é somente nutrir o RN, mas também promover uma interação entre mãe e filho. Amamentar faz bem à saúde da mãe, do bebê e do planeta. ${ }^{1,2}$

O AM contribui com o meio ambiente, pois, ao contrário dos leites artificiais, não agride e nem polui, além de já ser naturalmente preparado na temperatura ideal para o bebê com todos os nutrientes necessários, sem gasto de energia, de maneira eficaz. A oferta de leites artificiais prejudica a criança quanto ao seu crescimento e desenvolvimento, podendo levá-la tanto à desnutrição quanto à obesidade..$^{3,4}$

Segundo a OMS e o Fundo das Nações Unidas para a Infância (UNICEF), o AME previne cerca de 6 milhões de mortes infantis por ano. Para a saúde do RN, o leite materno (LM) traz vários benefícios, tais como: proteção contra infecções gastrointestinais, respiratórios e desnutrição. Estima-se que o LM pode prevenir $72 \%$ das internações infantis causadas por diarreia e $57 \%$ daquelas causadas por infecções respiratórias. ${ }^{5,6}$

O AM também traz benefícios para a saúde da mãe, reduzindo a incidência de câncer de mama, cânceres ovarianos, diabetes e fraturas ósseas por osteoporose. Proporciona uma involução uterina mais rápida, diminuindo o sangramento pós-parto e, consequentemente, colaborando para um menor quadro anêmico. Amplia os intervalos intergestacionais, pois sua eficácia como contraceptivo natural é de $98 \%$ nos seis primeiros meses após o parto, desde que a mulher esteja amamentando exclusivamente e se mantendo amenorreica. ${ }^{7-10}$

Com o intuito de manter o AME, a Constituição Federal de 5 de outubro de 1988 garante a proteção ao AM com a licença maternidade de 120 dias, sem prejuízo do emprego e salário. Em 2008, a partir da promulgação da Lei no 11.770 , de 9 de setembro, o benefício de 120 dias pode ser prolongado de forma facultativa para 180 dias, acordado entre sindicatos de empresas e empregados, podendo se dar tanto para trabalhadoras da esfera privada quanto para as de esfera pública. 2,10,11

Entretanto, ainda hoje no Brasil, o número de crianças que recebem AME até os 6 meses de idade está muito abaixo do que preconiza o UNICEF, da OMS e do MS, o que torna a situação preocupante. Dados da II Pesquisa de Prevalência do AM nas capitais brasileiras e Distrito Federal, realizada em 2008, apontaram que apenas $41,0 \%$ das crianças até 6 meses de vida recebiam AME. ${ }^{12,13}$ 
Tendo em vista que o desmame precoce do AME é um problema presente em todos os municípios brasileiros, a realização desse estudo se faz necessária, por oferecer informações sobre os motivos que levam ao desmame precoce, contribuindo para mudanças na prática profissional e também para o envolvimento e comprometimento dos gestores do serviço de saúde em elaborarem estratégias com o objetivo de incentivar o AM, contribuindo para melhoria da qualidade da alimentação das crianças e também para a prevenção dos agravos decorrentes da ausência desse aleitamento.

Nessa linha de pensamento, o estudo objetivou investigar os fatores relacionados ao desmame precoce do aleitamento materno exclusivo antes dos seis meses de vida.

\section{Métodos}

Trata-se de uma pesquisa de cunho exploratório, descritiva de abordagem quantitativa. Classifica-se como quantitativa, pois os resultados foram analisados buscando a opinião de mães de crianças com idade entre 0 a 6 meses em relação ao desmame precoce, de acordo com a frequência e o percentual das respostas. É exploratória por identificar os problemas relacionados ao desmame precoce. Tem finalidade descritiva pelo intuito de descrever as opiniões de um grupo de mulheres pertencentes às Estratégias de Saúde da Família (ESF).

A pesquisa foi realizada no município de Santo Antônio do Monte, situado no centro-oeste de Minas Gerais. O município possui uma população 27.938 habitantes, segundo dados do Instituto Brasileiro de Geografia e Estatística (IBGE), de 2016. Além de uma Santa Casa de Misericórdia e uma Unidade de Pronto Atendimento (UPA) 24h possui nove ESF, sendo seis urbanas e três rurais.

O grupo pesquisado foi composto por mulheres com filhos de 0 a 6 meses de idade pertencentes às ESFs urbanas. De acordo com o Departamento de Informática do Sistema único de Saúde (DATASUS), nasceram no município, nos últimos seis meses, cerca de 120 crianças. Foram incluídas as mães de crianças que desejaram participar do estudo, maiores de 18 anos de idade, e que não se encontravam em AME.

As ESFs rurais foram excluídas da amostra devido ao difícil acesso. A abordagem às participantes aconteceu nas unidades de saúde, anteriormente às consultas de puericultura e, quando não possível, foi feita em domicílio, contatado previamente por telefone. A aplicação do questionário realizou-se em ambiente iluminado, privado de ruídos e sem extravios de informações.

A coleta de dados foi realizada por meio de um questionário semiestruturado baseado no estudo de Farias e Wisniewski, ${ }^{14}$ aplicado face a face às entrevistadas, contendo perguntas objetivas que buscavam delinear um perfil melhor e mais abrangente sobre o grupo pesquisado, tais como: idade, escolaridade, estado civil, número de gestações, quantidade de filhos, bem como compreender os fatores que as levaram ao desmame precoce do AME. Nesse sentido, por ser semiestruturado, o questionário já continha algumas possíveis causas para o desmame mais apontadas em outros estudos, de acordo com a revisão bibliográfica sobre o tema feita anteriormente.

Os dados foram processados no programa estatístico Statistical Package for the Social Sciences (SPSS) 20.0. Para a análise descritiva dos dados, foi realizada a distribuição de frequência das variáveis, sendo estes dados apresentados em tabelas. Os resultados do estudo serão entregues, em forma de relatório, à Secretaria Municipal de Saúde para que as informações geradas possam subsidiar ações de incentivo ao AM. 
A pesquisa obedeceu às normas contidas na resolução 466/12 do Conselho Nacional de Saúde que dispõe sobre pesquisa envolvendo seres humanos. As participantes assinaram o Termo de Livre Consentimento Esclarecido (Apêndice A) e foram informadas dos objetivos da pesquisa e comunicadas que poderiam retirar seu nome em qualquer momento. O projeto foi submetido ao Comitê de Ética em Pesquisa UEMG/Divinópolis e aprovado sob o parecer de nํㅜ 2.086.812.

\section{Resultados}

Dentre as 120 mães de crianças menores de 6 meses, 82 não estavam realizando AME, porém apenas 52 corresponderam aos demais critérios de inclusão da pesquisa, Sendo assim, das mães entrevistadas, $42 \%$ apresentavam idade entre 18 e 23 anos de idade, de etnia branca (67\%), com escolaridade até o Ensino Médio (37\%). Constatou-se que 48\% tinham ocupação domiciliar e 48\% trabalhavam fora de casa em emprego formal. Todas definiram sua renda familiar de 1 a 2 salários mínimos. Mais da metade das entrevistadas (54\%) relatou ser casada, conforme pode ser observado na Tabela 1.

Tabela 1. Características socioeconômicas das mães entrevistadas em Santo Antônio do Monte/MG, 2017.

\begin{tabular}{|c|c|c|}
\hline Características & $\mathbf{N}$ & $\%$ \\
\hline \multicolumn{3}{|l|}{ Idade materna (anos) } \\
\hline 18 a 23 & 22 & 42 \\
\hline 24 a 28 & 18 & 35 \\
\hline 29 a 33 & 12 & 23 \\
\hline \multicolumn{3}{|l|}{ Etnia } \\
\hline Branca & 35 & 67 \\
\hline Não branca & 17 & 33 \\
\hline \multicolumn{3}{|l|}{ Escolaridade } \\
\hline Fundamental incompleto & 15 & 29 \\
\hline Fundamental completo & 9 & 17 \\
\hline Médio incompleto & 9 & 17 \\
\hline Médio completo & 19 & 37 \\
\hline \multicolumn{3}{|l|}{ Ocupação } \\
\hline Domiciliar & 25 & 48 \\
\hline Trabalha fora com carteira assinada & 25 & 48 \\
\hline Trabalha fora sem carteira assinada & 2 & 4 \\
\hline \multicolumn{3}{|l|}{ Renda (salário mínimo) } \\
\hline 1 a 2 & 52 & 100 \\
\hline \multicolumn{3}{|l|}{ Estado civil } \\
\hline Casada & 28 & 54 \\
\hline Solteira & 24 & 46 \\
\hline
\end{tabular}


As entrevistadas tiveram em média 1 filho (48\%), que não foi planejado (60\%), sendo que todas relataram ter realizado as consultas de pré-natal e mais que 8 consultas (75\%). Quanto ao AME, 40\% das mães entrevistadas afirmaram ter amamentado seu filho até os três meses de vida, e que deixaram de amamentar devido seu leite ser fraco ou insuficiente para o bebê (31\%). Embora uma porcentagem considerável, $27 \%$, tenha associado o descontinuamente do AME à volta ao trabalho fora de casa (Tabela 2).

Tabela 2. Caracterização do perfil gestacional e da amamentação das mães entrevistadas em em Santo Antônio do Monte/MG, 2017.

\begin{tabular}{|c|c|c|}
\hline Características & $\mathbf{N}$ & $\%$ \\
\hline \multicolumn{3}{|l|}{ Número de filhos } \\
\hline 1 & 25 & 48 \\
\hline 2 a 3 & 23 & 44 \\
\hline 4 a 5 & 4 & 8 \\
\hline \multicolumn{3}{|l|}{ Filho planejado } \\
\hline Sim & 21 & 40 \\
\hline Não & 31 & 60 \\
\hline \multicolumn{3}{|l|}{ Pré-natal } \\
\hline Sim & 52 & 100 \\
\hline \multicolumn{3}{|l|}{ Número de consultas de pré-natal } \\
\hline 7 a 8 & 12 & 23 \\
\hline Mais que 8 & 39 & 75 \\
\hline 4 a 6 & 1 & 2 \\
\hline \multicolumn{3}{|l|}{ Aleitamento materno exclusivo (em meses) } \\
\hline Menos que 1 & 18 & 35 \\
\hline 1 a 3 & 21 & 40 \\
\hline 4 a 5 & 13 & 25 \\
\hline \multicolumn{3}{|l|}{ Motivo que deixou o AME antes dos 6 meses de idade } \\
\hline Leite fraco ou insuficiente para o bebê & 16 & 31 \\
\hline Precisar voltar ao trabalho & 14 & 27 \\
\hline Bebê chorar e não pegar & 10 & 19 \\
\hline Não aceitava o peito & 8 & 15 \\
\hline Problemas nas mamas (rachaduras) & 1 & 2 \\
\hline Substituição do LM por produtos industrializados & 1 & 2 \\
\hline Crenças, culturas e estilo de vida (queda dos seios) & 1 & 2 \\
\hline Influência de terceiros & 1 & 2 \\
\hline
\end{tabular}

AME: aleitamento materno exclusivo; LM: leite materno.

A Tabela 3 demonstra que $92 \%$ das mães receberam informações de profissionais de saúde sobre o AME e o profissional que mais as orientou foi o enfermeiro (77\%). Metade das entrevistadas disseram não ter sido orientadas a dar outro leite para o bebê antes dos 6 meses de idade. Em relação às orientações recebidas, $77 \%$ afirmaram as ter recebido do profissional médico, sendo que $58 \%$ concordaram que receber orientações do serviço de saúde as facilitaria a amamentar por maior período de tempo. Importa destacar nesse quesito que poucas mães associaram a importância do apoio familiar para a continuidade do AME. 
Tabela 3. Caracterização das orientações advindas dos profissionais de saúde definidas pelas mães entrevistadas em Santo Antônio do Monte/MG, 2017.

\begin{tabular}{|c|c|c|}
\hline Características & $\mathbf{N}$ & $\%$ \\
\hline \multicolumn{3}{|c|}{ Orientações dos profissionais de saúde sobre AME } \\
\hline $\operatorname{Sim}$ & 48 & 92 \\
\hline Não & 4 & 8 \\
\hline \multicolumn{3}{|c|}{ Profissionais que orientaram quanto ao AME até os 6 meses de idade } \\
\hline Enfermeiro & 40 & 77 \\
\hline Médico & 12 & 23 \\
\hline \multicolumn{3}{|c|}{ Orientada a dar outro leite para o bebê antes dos 6 meses de idade } \\
\hline Sim & 26 & 50 \\
\hline Não & 26 & 50 \\
\hline \multicolumn{3}{|l|}{ Quem a orientou } \\
\hline Enfermeiro & 1 & 4 \\
\hline Médico & 20 & 77 \\
\hline Avós & 3 & 11 \\
\hline Outros & 2 & 8 \\
\hline \multicolumn{3}{|l|}{ O que a facilitaria a amamentar } \\
\hline Orientações do serviço de saúde & 30 & 58 \\
\hline Não trabalhar fora & 12 & 23 \\
\hline Já ter amamentado antes & 9 & 17 \\
\hline Apoio familiar & 1 & 2 \\
\hline
\end{tabular}

\section{Discussão}

No estudo aqui discutido, observou-se a maior ocorrência do desmame precoce com mulheres mais jovens. Este fato pode se dar por elas serem menos experientes, possuírem mais dúvidas e anseios relacionados ao AM. Resultados semelhantes foram encontrados em outros estudos, em que as mães também eram jovens. ${ }^{15}$ Infere-se, portanto, que a pouca idade materna afeta diretamente a manutenção do AM apresentando uma tendência a desmamar precocemente seus bebês.

A prevalência do desmame precoce quanto à etnia foi observada em maior número em mães brancas. Porém, um estudo ${ }^{16}$ afirma estudo que mães não brancas estão mais predispostas a interromper o AME de seus filhos quando comparadas às mães brancas. Portanto, este fator permanece contraditório e devido à escassez de estudos sobre o tema torna-se difícil saber se este fato está relacionado ou não com a interrupção precoce do AM, necessitando de mais estudos para validar a questão.

Em relação ao grau de escolaridade, mães com menor escolaridade tendem a desmamar seus filhos precocemente. Isso pode ocorrer devido ao menor acesso às informações a respeito das vantagens do AM em comparação às mães com maior nível educacional. As mães de menor renda costumam amamentar por menos tempo, por procurarem com baixa frequência o serviço de saúde. Mães com maior renda familiar apresentaram maior conhecimento sobre aleitamento. ${ }^{17,18}$ 
Um dado relevante na presente pesquisa foi a situação conjugal, mais da metade das entrevistadas tinha companheiro. O apoio familiar é de grande relevância para as nutrizes, pois contribui para a alta eficácia da amamentação. Os familiares devem estar envolvidos neste processo, pois para a mãe é um momento novo que apresenta muitos medos, dificuldades e insegurança, que requer total apoio e incentivo familiar. ${ }^{19}$

Conjugado a isso, há o fato das nutrizes terem ocupação domiciliar, o que pode influenciar o desmame precoce devido à sobrecarga do trabalho doméstico. Com a dedicação ao lar, os filhos acabam sendo deixados em segundo plano e não recebem a atenção necessária, ou seja, elas não têm tempo para se dedicar ao AME.

O processo de amamentar é um processo interativo com a família e o cotidiano social. Faz-se importante que maridos ou demais familiares participem deste processo providenciando os cuidados diários, enquanto as mães amamentam. Estudos apontam a importância de agregar familiares nas atividades de atenção à saúde para melhor apoio à nutriz.,8

Em relação à ocupação das mães, a questão delas trabalharem fora de casa em emprego formal também pode favorecer à interrupção precoce do AME. Especialistas afirmam que mesmo havendo Políticas de Apoio à Mulher Trabalhadora que Amamenta nem sempre elas abordam de maneira satisfatória as condições de trabalho da mulher e não contribuem algumas vezes com a manutenção da amamentação além da licença maternidade. ${ }^{20}$

Alguns fatores que podem contribuir para essa situação foram enumerados em uma pesquisa. ${ }^{21}$ Muitas vezes, o local de trabalho da nutriz é inadequado para o $A M$, podendo haver constrangimentos quanto à exposição do seio ao amamentar em público, incerteza e preocupações com o ganho de peso do bebê, dificuldade de praticar a ordenha antes do retorno ao trabalho, condições insalubres para a ordenha do leite no local de trabalho, falta de informação e interesse da empresa quanto às políticas de AM, cansaço da mãe, entre outros.

O desmame precoce do AM se mostrou mais elevado em mães que possuíam apenas um filho. Este fato pode ser explicado pela baixa experiência e imaturidade para cuidar e amamentar seus filhos. Destarte, é importante uma maior adesão, por parte das nutrizes, às ações de aconselhamento sobre o aleitamento, principalmente para as primíparas. Estas ações devem partir preferencialmente da Unidade Básica de Saúde (UBS), tendo como foco principal a promoção do AM e prevenção de agravos decorrentes da interrupção desta prática antes do preconizado pelo MS. ${ }^{22}$

Observou-se maior prevalência do desmame precoce entre mães que não tiveram a gravidez planejada. Isso pode acontecer pelo fato de não ser uma gravidez esperada e a mãe não estar preparada para dedicar-se aos cuidados com o filho, tendo dificuldades em amamentar. Esta questão deve ser um alerta para os profissionais de saúde para que apoiem e incentivem estas mães de modo que seja estabelecido o vínculo com o bebê e o processo do AME seja alcançado com sucesso. É importante orientar, acolher e acompanhar esta nutriz individualmente a fim de obter uma melhoria da média do AME. ${ }^{14,23}$

Um paradoxo percebido no estudo foi o fato de todas as mães terem afirmado que realizaram as consultas de pré-natal periodicamente, e ao mesmo tempo um número considerável de mães interromperam 
precocemente o AME. Essa questão evidencia que, nem sempre, as informações transmitidas nas consultas podem ser de boa qualidade, que não foram discutidas durante as consultas de pré-natal sobre a amamentação ou então, que os usuários não fazem bom uso dessas informações. A boa comunicação é fundamental para a eficácia do processo da amamentação. ${ }^{8}$

Para além da boa comunicação, a produção de vínculo com a população dos territórios de saúde possibilita a longitudinalidade do cuidado e o alcance de seus benefícios. O fortalecimento do vínculo por meio de uma atenção singular, individualizada e humanizada, estimula a confiança e a construção da autonomia por parte dos usuários, facilitando a adesão às orientações educativas propostas pelos profissionais de saúde. ${ }^{24-26}$

Notou-se que entre o quarto e quinto mês de vida da criança houve menor adesão ao AME. Entre o quinto e sexto mês pode haver uma queda significativa do AME devido à introdução de outros leites e alimentos sólidos. ${ }^{14}$ Cabe ao profissional desencorajar as mães quanto à introdução precoce de novos alimentos, a fim de melhorar a qualidade de vida do RN. ${ }^{4}$

Quanto aos fatores que as influenciaram ao desmame precoce, a crença de que o LM seria fraco ou insuficiente para o bebê se destacou. Muitas nutrizes pensam desta maneira pelo fato de, em algumas ocasiões, o LM se apresentar transparente ou com aspecto semelhante à água de coco.

Esse pensamento pode estar associado à falta de conhecimento da fisiologia do LM, ou a fatores culturais, que interferem fortemente nas crenças maternas, mesmo que as mães tenham sido orientadas corretamente pelos profissionais de saúde. Pode-se considerar também que algumas não queiram amamentar, porém não assumem para si próprias que deixaram de amamentar sem motivo e utilizam este fato como uma justificativa para si mesma e para os que estão em sua volta. ${ }^{15,27}$

Outra justificativa para a interrupção precoce do AME foi a questão de o bebê chorar e não pegar o seio. Essa resistência da criança pode ser devido à introdução de outros bicos artificiais ou mamadeiras, surgimento de dor ao ser posicionado em cada mamada ou a pega inadequada ao seio. Estas determinadas situações podem ser prevenidas com orientações dos profissionais de saúde, os quais devem estar atentos durante todo o período da amamentação. ${ }^{2,27}$

Pode-se notar um dado satisfatório quanto às orientações recebidas pelas mães por meio dos profissionais de saúde em relação ao AM, principalmente por parte do profissional enfermeiro. O profissional de saúde desde a primeira consulta de pré-natal, no nascimento, no pós-parto, puericultura, assim como nas imunizações, deve incentivar, promover e apoiar o AM. O profissional deve escutar atentamente suas dúvidas, compreender, estimular a autoconfiança e auxiliá-las a enfrentar todas as dificuldades pré-estabelecidas, orientando-as quanto a uma prática saudável do AM. ${ }^{28}$

Em relação à introdução de outros tipos de leite antes dos seis meses de idade, os profissionais médicos são favoráveis ao $\mathrm{AM},{ }^{29}$ no entanto, um estudo realizado em um hospital municipal de São Paulo evidenciou que os médicos devem ter uma visão diferenciada sobre a introdução de complementação ao aleitamento materno porque recebem capacitação específica. ${ }^{15}$

O recomendado é que não seja dado ao RN nenhum outro alimento ou bebida além do LM, mas faz a ressalva de que o complemento seja com indicação médica. Entretanto, os médicos não estão 
envolvidos com os acontecimentos rotineiros da nutriz e RN. Determinadas vezes, não conhecem a fundo as dificuldades da mãe, ou seja, não ouvem a queixa da nutriz para investigar o fato, optando assim pela iniciação de suplementos, como as fórmulas infantis, induzindo, desta forma, o desmame precoce por algo que talvez poderia ser resolvido por meio de orientações.

Nesse estudo, segundo as entrevistadas, constatou-se que o que as facilitaria a amamentar por mais tempo seria receber mais orientações do serviço de saúde. Embora o processo de amamentar seja natural, ele envolve um desenrolar de mudanças para a mulher, tanto psicologicamente quanto fisiologicamente, o que requer muito apoio, principalmente por parte do serviço de saúde que saberá orientá-las corretamente..$^{30}$

De acordo com as entrevistadas, outra forma que as facilitaria quanto à maior durabilidade da amamentação seria elas não trabalharem fora de casa, pois o fim da licença maternidade coincide com o término do período de AME ou, dependendo da empresa, até mesmo antes, com 120 dias, tendo estas mães que retornar ao trabalho enquanto seus filhos tiverem apenas quatro meses de vida, perdendo assim a eficácia da amamentação. ${ }^{31}$ Dessa forma, pensar em políticas trabalhistas que incentivem a AME, aumentando o tempo de licença maternidade ou ainda favorecendo o acolhimento das nutrizes e lactentes, ao proporcionar ambientes adequados para a amamentação e/ou ordenha.

A questão de as mães já terem amamentado anteriormente, segundo as entrevistadas, também é um ponto facilitador. As mães que já possuem maior experiência sobre a amamentação apresentam maior autoeficácia e são determinantes importantes das altas taxas de AM. ${ }^{32}$

\section{Conclusões}

Diante desse estudo, verificou-se que os principais fatores determinantes ao desmame precoce foram a crença quanto ao leite fraco ou insuficiente para o bebê, retorno ao trabalho, má interpretação do choro e inexperiência ou insegurança da mãe. Embora não tenha sido um fator determinante para o problema pesquisado, a atuação/orientação profissional se destacou no estudo. O sucesso da amamentação depende primeiramente da vontade da mãe de amamentar e, posteriormente, da atuação do profissional de saúde, do apoio familiar e de condições favoráveis no local de trabalho.

A partir dos resultados apontados o profissional de saúde, juntamente à classe empregadora podem elaborar estratégias e ações educativas priorizando ainda mais a importância do AM e ter maior resolutividade quanto aos fatores destacados. Por ser uma questão ampla, o profissional não deve apenas priorizar os fatores biológicos, mas também buscar melhoria no vínculo com a gestante, valorizar questões de cunho psicológico e social, apoiando e orientando ainda mais a nutriz.

A diversidade de pesquisas encontradas quanto ao tema mostra a gravidade e a baixa incidência do AM, tornando-o assim um problema de saúde pública. Salienta-se a importância da implementação de estratégias que visem a adesão, promoção, manutenção e aumento da prevalência do AM.

Espera-se que os achados desse estudo possam servir de subsídios para futuras avaliações dos serviços de saúde em relação ao AM, contribuindo, assim, para a redução das taxas de morbidade e mortalidade infantil. 


\section{Referências}

1. Souza NKT, Medeiros MP, Silva MA, Cavalcanti SB, Dias RS, Valente FA. Aspectos envolvidos na interrupção do aleitamento materno exclusivo. Comun Ciênc Saúde. 2011;22(4):231-8.

2. Brasil. Ministério da Saúde. Secretaria de Atenção à Saúde. Departamento de Atenção Básica. Cadernos de Atenção Básica. Saúde da criança: aleitamento materno e alimentação complementar. Brasília: Ministério da Saúde; 2015.

3. Queluz MC, Pereira MJB, Santos CB, Leite AM, Ricco RG. Prevalência e determinantes do aleitamento materno exclusivo no município de Serrana, São Paulo, Brasil. Rev Esc Enferm USP. 2012;46(3):537-43. http://dx.doi.org/10.1590/S0080-62342012000300002

4. Monteschio CAC, Gaiva MAM, Moreira MDS. O enfermeiro frente ao desmame precoce na consulta de enfermagem à criança. Rev Bras Enferm. 2015;68(5):869-75. http://dx.doi.org/10.1590/0034-7167.2015680515i

5. Sassa AH, Schmidt KT, Rodrigues BC, Ichisato SMT, Higarashi IH, Marcon SS. Bebês pré-termo: aleitamento materno e evolução ponderal. Rev Bras Enferm. 2014;67(4):594-600. http://dx.doi.org/10.1590/0034-7167.2014670415

6. Victora CG, Barros AJD, França GVA, Bahl R, Rollins NC, Horton S, et al. Amamentação no século 21: epidemiologia, mecanismos, e efeitos ao longo da vida. Epidemiol Serv Saúde. 2016;25(1):1-24.

7. Oliveira CS, locca FA, Carrijo MLR, Garcia RATM. Amamentação e as intercorrências que contribuem para o desmame precoce. Rev Gaúcha Enferm. 2015;36(n spe):16-23. http://dx.doi.org/10.1590/1983-1447.2015.esp.56766

8. Prado CVC, Fabbro MRC, Ferreira GI.Desmame precoce na perspectiva de puérperas: uma abordagem dialógica. Texto Contexto Enferm. 2016;25(2):e1580015.

9. Maranhão TA, Gomes KRO, Nunes LB, Moura LNB. Fatores associados ao aleitamento materno exclusivo entre mães adolescentes. Cad Saúde Coletiva. 2015;23(2):132-9. http://dx.doi.org/10.1590/1414-462X201500020072

10. Brasileiro AA, Ambrosano GMB, Marba STM, Possobon RF. A amamentação entre filhos de mulheres trabalhadoras. Rev Saúde Pública. 2012;46(4):642-8. http://dx.doi.org/10.1590/S0034-89102012005000053

11. Demetrio F, Pinto EJ, Assis AMO. Fatores associados à interrupção precoce do aleitamento materno: um estudo de coorte de nascimento em dois municípios do Recôncavo da Bahia, Brasil. Cad Saúde Pública. 2012;28(4):641-50. http://dx.doi.org/10.1590/ S0102-311X2012000400004

12. Oliveira MGOA, Lira PIC, Batista Filho M, Lima MC. Fatores associados ao aleitamento materno em dois municípios com baixo índice de desenvolvimento humano no Nordeste do Brasil. Rev Bras Epidemiol. 2013;16(1):178-89. http://dx.doi.org/10.1590/S1415$790 \times 2013000100017$

13. Barbosa LN, Santos NC, Moraes MAM, Rizzardi SD, Corrêa EC. Prevalência de práticas educativas acerca do aleitamento materno exclusivo (AME) em Cuiabá - MT. Esc Anna Nery. 2015;19(1):147-53.

14. Farias SE, Wisniewsk D. Aleitamento materno x desmame precoce. Rev Uningá. 2015;22(1):14-9.

15. Rocci E, Fernandes RAQ. Dificuldades no aleitamento materno e influência no desmame precoce. Rev Bras Enferm. 2014;67(1):22-7.

16. Moraes BA, Gonçalves AC, Strada JKR, Gouveia HG. Fatores associados à interrupção do aleitamento materno exclusivo em lactentes com até 30 dias. Rev Gaúcha Enferm. 2016;37(spe):e2016-0044. http://dx.doi.org/10.1590/1983-1447.2016.esp.2016-0044

17. Gusmao AM, Béria JU, Gigante LP, Leal AF, Schermann LB. Prevalência de aleitamento materno exclusivo e fatores associados: estudo transversal com mães adolescentes de 14 a 16 anos em Porto Alegre, RS, Brasil. Ciênc Saúde Coletiva. 2013;18(11):3357-68. http://dx.doi.org/10.1590/S1413-81232013001100025

18. Boff ADG, Paniagua LM, Scherer S, Goulart BNG. Aspectos socioeconômicos e conhecimento de puérperas sobre o aleitamento materno. Audiol Commun Res. 2015;20(2):141-5. http://dx.doi.org/10.1590/S2317-64312015000200001517

19. Rodrigues AP, Padoin SMM, Paula CC, Guido LA. Fatores que interferem na autoeficácia da amamentação: revisão integrativa. Rev Enferm UFPE on line [publicação na Internet]. 2013 [citado 13 Dez 2017] 7(spe):4144-52. Disponível em: http://www.revista.ufpe.br/ revistaenfermagem/index.php/revista/article/download/4031/6315.

20. Kalil IR, Aguiar AC. Trabalho feminino, políticas familiares e discursos pró-aleitamento materno: avanços e desafios à equidade de gênero. Saúde Debate. 2016;40(110):208-23. 
21. Silva CA, Davim RMB. Mulher trabalhadora e fatores que interferem na amamentação: revisão integrativa. Rev RENE. 2012;13(5):1208-17.

22. Schincaglia RM, Oliveira AC, Sousa LM, Martins KA. Práticas alimentares e fatores associados à introdução precoce da alimentação complementar entre crianças menores de seis meses na região noroeste de Goiânia. Epidemiol Serv Saúde. 2015;24(3):465-74. http:// dx.doi.org/10.5123/S1679-49742015000300012

23. Conceição SP, Fernandes RAQ. Influência da gravidez não planejada no tempo de aleitamento materno. Esc Anna Nery.2015;19(4):600-5.

24. Baratieri T, Mandu ENT, Marcon SS. Compreensão de enfermeiros sobre vínculo e longitudinalidade do cuidado na Estratégia Saúde da Família. Ciênc Enferm. 2012;18(2):11-22. http://dx.doi.org/10.4067/S0717-95532012000200002

25. Bernardes AG, Pelliccioli EC, Marques CF. Vínculo e práticas de cuidado: correlações entre políticas de saúde e formas de subjetivação. Ciênc Saúde Coletiva. 2013;18(8):2339-46. http://dx.doi.org/10.1590/S1413-81232013000800018

26. Reichert APS, Rodrigues PF, Albuquerque TM, Collet N, Minayo MCS. Vínculo entre enfermeiros e mães de crianças menores de dois anos: percepção de enfermeiros. Ciênc Saúde Coletiva. 2016; 21(8):2375-82. http://dx.doi.org/10.1590/1413-81232015218.07662016

27. Amaral LJX, Sales SS, Carvalho DPSRP, Cruz GKP, Azevedo IC, Ferreira Júnior MA. Fatores que influenciam na interrupção do aleitamento materno exclusivo em nutrizes. Rev Gaúcha Enferm. 2015;36(spe):127-34. http://dx.doi.org/10.1590/1983-1447.2015.esp.56676

28. Almeida JM, Luz SAB, Ued FV. Apoio ao aleitamento materno pelos profissionais de saúde: revisão integrativa da literatura. Rev Paul Pediatr. 2015;33(3):355-62. http://dx.doi.org/10.1016/j.rpped.2014.10.002

29. Escarce AG, Araújo NG, Friche AAL, Motta AR. Influência da orientação sobre aleitamento materno no comportamento das usuárias de um hospital universitário. Rev CEFAC. 2013;15(6):1570-82. http://dx.doi.org/10.1590/S1516-18462013000600020

30. Batista KRA, Farias MCAD, Melo WSN. Influência da assistência de enfermagem na prática da amamentação no puerpério imediato. Saúde Debate. 2013;37(96):130-8. http://dx.doi.org/10.1590/S0103-11042013000100015

31. Barbieri MC, Bercini LO, Brondani KJM, Ferrari RAP, Tacla MTGM, Sant'anna FL. Aleitamento materno: orientações recebidas no pré-natal, parto e puerpério. Semina: Ciênc Biol Saúde. 2015;36(1 Suppl):17-24.

32. Sirio MAO, Freitas SN, Figueiredo AM, Gouvêa GDR, Pena JL, Machado-Coelho GLL. Tempo de aleitamento materno entre indígenas Xakriabá aldeados em Minas Gerais, Sudeste do Brasil. Rev Nutr.2015;28(3):241-52. http://dx.doi.org/10.1590/1415-52732015000300002 